\title{
CONHECIMENTOS DE ADOLESCENTES SOBRE MÉTODOS CONTRACEPTIVOS E INFECÇÕES SEXUALMENTE TRANSMISSÍVEIS
}

\author{
ADOLESCENTS' KNOWLEDGE ABOUT \\ CONTRACEPTIVE METHODS AND SEXUALLY \\ TRANSMITTED INFECTIONS
}

\section{CONOCIMIENTO DE LOS ADOLESCENTES SOBRE MÉTODOS ANTICONCEPTIVOS E INFECCIONES DE TRANSMISIÓN SEXUAL}

\author{
Kléber José Vieira ${ }^{1}$ \\ Nayara Gonçalves Barbosa ${ }^{2}$ \\ Juliana Cristina dos Santos Monteiro ${ }^{3}$ \\ Letícia de Almeida Dionízio ${ }^{4}$ \\ Flávia Azevedo Gomes-Sponholz
}

Como citar este artigo: Vieira KJ, Barbosa NG, Monteiro JCS, Dionízio LA, Gomes-Sponholz FA. Conhecimentos de adolescentes sobre métodos contraceptivos e infecções sexualmente transmissíveis. Rev baiana enferm. 2021;35:e39015.

Objetivos: identificar os conhecimentos de adolescentes sobre práticas sexuais seguras e identificar as necessidades de informação dos adolescentes sobre infecções sexualmente transmissíveis e gravidez. Método: estudo transversal realizado com 499 adolescentes de escola pública, de fevereiro a abril de 2017, por meio de questionário autoaplicado e semiestruturado. Resultados: o método mais conhecido foi o preservativo masculino ( $94,4 \%)$; dentre os adolescentes do sexo masculino, 22,7\% julgaram desnecessário o uso de preservativo em todas as relações sexuais $(\mathrm{p}<0,01)$ e $24,6 \%$ afirmaram que contraceptivos orais protegiam contra infecções sexualmente transmissíveis $(\mathrm{p}=0,04)$. Conclusão: os adolescentes apresentaram concepções prévias e eventualmente superficiais a respeito da prevenção de IST e da gravidez. As participantes do sexo feminino apresentaram maior conhecimento a respeito dos métodos contraceptivos, infecções sexualmente transmissíveis e práticas de sexo seguro. A identificação de deficiência no conhecimento apresentada pelo grupo investigado recomenda a realização de atividades relacionadas ao tema da educação sexual nas escolas.

Descritores: Saúde do Adolescente. Educação Sexual. Gravidez na Adolescência. Infecções Sexualmente Transmissíveis.

Objectives: to identify adolescents' knowledge of safe sexual practices and to identify adolescents' information needs about sexually transmitted infections and pregnancy. Method: cross-sectional study conducted with 499 adolescents in public school, from February to April 2017, by using a self-applied and structured questionnaire. Results: the best

\footnotetext{
Assistente Social. Mestre em Enfermagem em Saúde Pública. Hospital Municipal Bela Vista. São Paulo, São Paulo, Brasil. klebervieiraseso@gmail.com. http://orcid. org/0000-0002-8151-1667.

2 Enfermeira. Doutora em Ciências. Professora da Escola de Enfermagem de Ribeirão Preto da Universidade de São Paulo. Ribeirão Preto, São Paulo, Brasil. nagbarbosa@gmail.com. http://orcid.org/0000-0003-3646-4133.

Enfermeira. Doutora em Saúde Pública. Professora Associada da Escola de Enfermagem de Ribeirão Preto da Universidade de São Paulo. Ribeirão Preto, São Paulo, Brasil. http://orcid.org/0000-000 I-6470-673X.

4 Enfermeira do Centro de Referência da Saúde da Mulher de Ribeirão Preto - MATER. Ribeirão Preto, São Paulo, Brasil. https://orcid.org/0000-0003-2236-6436.

5 Enfermeira. Doutora em Saúde Pública. Professora Associada da Escola de Enfermagem de Ribeirão Preto da Universidade de São Paulo. Ribeirão Preto, São Paulo, Brasil. https://orcid.org/0000-0003-1540-0659.
} 
known method was the male condom (94.4\%); among male adolescents, $22.7 \%$ considered condom use unnecessary in all sexual relations $(p<0.01)$ and $24.6 \%$ stated that oral contraceptives protected against sexually transmitted infections ( $p=0.04$ ). Conclusion: the adolescents presented previous and eventually superficial conceptions regarding the prevention of STI and pregnancy. Female participants presented greater knowledge about contraceptive methods, sexually transmitted infections and safe sex practices. The identification of disabilities in the knowledge presented by the group recommends activities related to sexual education in schools.

Descriptors: Adolescent Health. Sex Education. Pregnancy in Adolescence. Sexually Transmitted Infections.

Objetivos: identificar los conocimientos de los adolescentes sobre las prácticas sexuales seguras e identificar las necesidades de información de los adolescentes sobre las infecciones de transmisión sexual y el embarazo. Método: estudio transversal realizado con 499 adolescentes de escuela pública, de febrero a abril de 2017, mediante un cuestionario autoadministrado y semiestructurado. Resultados: el método más conocido era el preservativo masculino (94,4\%); entre los adolescentes del sexo masculino, el 22, 7\% consideraba innecesario el uso del preservativo en todas las relaciones sexuales $(p<0,01)$ y el $24,6 \%$ afirmaba que los anticonceptivos orales protegian contra las infecciones de transmisión sexual $(p=0,04)$. Conclusión: los adolescentes presentaban concepciones previas y posiblemente superficiales sobre la prevención de las ITS y el embarazo. Las participantes femeninas presentaban un mayor conocimiento sobre los métodos anticonceptivos, las infecciones de transmisión sexual y las prácticas sexuales más seguras. La identificación de discapacidades en los conocimientos presentados por el grupo recomienda actividades relacionadas con el tema de la educación sexual en las escuelas.

Descriptores: Salud del Adolescente. Educación Sexual. Embarazo en Adolescencia. Infecciones de Transmisión Sexual.

\section{Introdução}

Nos últimos anos, devido ao desenvolvimento tecnológico e de inteligência artificial, houve um aumento na disponibilidade de informações de conteúdo sexual na internet ${ }^{(1)}$. Apesar do maior acesso à informação, o déficit de conhecimento dos adolescentes a respeito dos métodos contraceptivos, da prevenção de infecções sexualmente transmissíveis (IST) e das questões sobre a sexualidade persiste e representa um problema atual e pertinente ${ }^{(2)}$.

Os adolescentes, apesar de sua habilidade em tecnologia, são vulneráveis no que diz respeito às escolhas das informações ofertadas livremente $^{(3)}$. Oriundo desse quadro deficitário, um evento recorrente e polêmico no cenário da saúde, o sexo inseguro, tem como principais desdobramentos a gravidez indesejada e/ou doenças de transmissão sexual, que envolvem os indivíduos, a família e a sociedade, além de aumentar os custos da atenção à saúde em todos os níveis de assistência ${ }^{(2)}$.

No Brasil, embora seja observada uma queda de 33\% na taxa de gravidez na adolescência no período de 2000 a 2016, os dados desse evento permanecem preocupantes. A taxa brasileira de gravidez na adolescência é de 58,7/1000, superior à taxa média das Américas, de 48,6/1000 ${ }^{(4)}$. Estima-se que aproximadamente dois terços das gestações em adolescentes menores de 18 anos não sejam intencionais ${ }^{(5)}$. Quanto ao desfecho, a gravidez na adolescência é a segunda maior causa de morte em mulheres no mundo e associa-se com o maior risco de pré-eclâmpsia, recém-nascidos pré-termo e de baixo peso e com a ocorrência de abortos e natimortos ${ }^{(6)}$.

Ao considerar os prejuízos à saúde dos adolescentes, decorrentes da falta de informação adequada e de qualidade, as IST constituem-se, da mesma forma que a gravidez não planejada, questão de saúde pública ${ }^{(2)}$. Os dados sobre prevalência de doenças sexualmente transmissíveis entre a população adolescente e jovem no Brasil são imprecisos. Destaca-se como um fator de exposição, a prática sexual sem proteção ou com uso não frequente de preservativos, seja entre parceiros estáveis, seja em relações eventuais ${ }^{(7)}$.

Nesse contexto, identificar o conhecimento que adolescentes têm sobre sexo seguro, prevenção de gestação e de infecções é necessário para que estratégias educativas, integrais e com 
informações seguras, além de fundamentadas em evidências científicas, possam ser implementadas. Destaca-se a recomendação do Ministério da Saúde de que as temáticas saúde sexual e reprodutiva e prevenção de IST sejam trabalhadas de forma educativa com os alunos das séries finais do ensino fundamental até médio ${ }^{(8)}$.

O presente estudo tem como objetivo identificar os conhecimentos de adolescentes sobre práticas sexuais seguras e identificar as necessidades de informação dos adolescentes sobre IST e gravidez.

\section{Método}

Desenvolveu-se um estudo exploratório, transversal, descritivo e de caráter quantitativo em uma escola pública do munícipio de Pouso Alegre, Minas Gerais, com dados coletados no período de fevereiro a abril de 2017.

O cenário do estudo foi a Escola Estadual Monsenhor José Paulino, que possui cerca de 1.250 alunos e funciona nos períodos matutino, vespertino e noturno. No total, 700 estudantes matriculados do $8^{\circ}$ ano do ensino fundamental ao $3^{\circ}$ ano do ensino médio foram convidados para participarem da pesquisa, dos quais 499 compuseram a amostra do estudo.

Os adolescentes foram incluídos na pesquisa mediante a assinatura do Termo de Consentimento Livre e Esclarecido, pelos pais ou responsáveis legais, e do Termo de Assentimento Livre e Esclarecido, pelo próprio participante. Os critérios de inclusão foram: estar matriculado e frequentar a escola, do $8^{\circ}$ ano do ensino fundamental ao $3^{\circ}$ ano do ensino médio. Foram excluídos os alunos com idade inferior a 10 anos e superior a 19 anos ou os que se evadiram da escola até a ocasião do estudo. O estudo foi aprovado por um Comitê de Ética e Pesquisa da Escola de Enfermagem de Ribeirão Preto, Universidade São Paulo, Certificado de Apresentação de Apreciação Ética (CAAE) número 62175616.3.0000.5393.

Utilizou-se como instrumento de coleta de dados um questionário autorrespondido, anônimo. O modelo usado no questionário foi pautado no documento intitulado "Pesquisa de Atitudes e Práticas da População Brasileira”(9). As variáveis avaliadas, em conformidade com a técnica de questionário autoaplicável, foram as seguintes: idade na menarca, familiaridade com métodos contraceptivos, participação em oficinas de educação sexual, discussão sobre a sexualidade com a família e amigos e desejo de ser mãe ou pai no futuro.

A análise dos dados coletados foi precedida pela elaboração de um banco, com a dupla digitação dos questionários no aplicativo Excel 2008, utilizando-se um dicionário de dados para a codificação das variáveis. Os dados foram analisados pelo programa estatístico Statistical Package for the Social Sciences (SPSS) versão 17.0.

\section{Resultados}

$\mathrm{Na}$ análise dos dados, emergiram três categorias que serão descritas a seguir: Caracterização dos adolescentes segundo variáveis demográficas, Conhecimento dos adolescentes sobre métodos contraceptivos e Informações sobre Infecções Sexualmente Transmissíveis (IST) e gravidez. Nestas são apresentados os resultados relativos à caracterização dos adolescentes segundo variáveis demográficas, conhecimento dos adolescentes sobre métodos contraceptivos e informações sobre IST e gravidez. Os dados também estão apresentados em tabelas.

\section{Caracterização dos adolescentes segundo variáveis demográficas}

A média da idade dos 499 participantes foi de $16,3$ anos (dp $\pm 1,7)$, variando de 12 a 17 anos. Aproximadamente um terço dos participantes 160 (32\%) - tinha entre 11 e 14 anos. Do total de participantes, 286 (57,3\%) pertenciam ao sexo feminino e $213(42,7 \%)$ ao sexo masculino. A maioria das participantes do sexo feminino, relatou menarca ( $\mathrm{N}=284 ; 99,3 \%)$. A idade, na menarca, variou de 8 a 16 anos, com média de 11,9 anos (dp $\pm 1,2)$. e mediana de 12 anos. 
Conhecimento dos adolescentes sobre métodos contraceptivos

Os principais métodos contraceptivos conhecidos pelos adolescentes entrevistados foram o preservativo masculino $(94,4 \%)$, o contraceptivo hormonal oral $(83,1 \%)$, o preservativo feminino (76,3\%) e a contracepção hormonal de emergência $(74,5 \%)$. Observou-se diferença quanto ao conhecimento dos métodos contraceptivos em relação ao sexo. As participantes do sexo feminino possuíam maiores informações quanto à diversidade de métodos contraceptivos, em destaque: preservativo feminino ( $\mathrm{p}<0,01)$, contraceptivo hormonal oral ( $\mathrm{p}<0,01)$, contracepção hormonal de emergência ( $\mathrm{p}<0,01)$, calendário ( $p<0,01)$ e laqueadura tubária $(\mathrm{p}<0,01)$ (Tabela 1).

Tabela 1 - Conhecimento dos adolescentes a respeito dos métodos contraceptivos. Pouso Alegre, Minas Gerais, Brasil - 2017

\begin{tabular}{lcc|c|c|c}
\hline Métodos & $\begin{array}{c}\text { Masculino } \\
\mathbf{n = 2 0 7}(\mathbf{\%})\end{array}$ & $\begin{array}{c}\text { Feminino } \\
\mathbf{n = 2 9 2}(\mathbf{\%})\end{array}$ & $\begin{array}{c}\text { Total } \\
\mathbf{n = 4 9 9 ( \% )}\end{array}$ & $\mathbf{p - v a l o r}$ & $\begin{array}{c}\text { Odds Ratio } \\
\text { (Minimo-Máximo) }\end{array}$ \\
\hline $\begin{array}{l}\text { Preservativo } \\
\text { masculino }\end{array}$ & $196(94,6)$ & $275(94,2)$ & $471(94,4)$ & $<0,75$ & $1,14(0,50-2,56)$ \\
Preservativo feminino & $140(67,6)$ & $241(82,5)$ & $381(76,3)$ & $<0,01^{*}$ & $0,43(0,28-0,66)$ \\
Hormonal oral & $154(74,4)$ & $261(89,4)$ & $415(83,1)$ & $<0,01^{*}$ & $0,33(0,20-0,54)$ \\
Pílula & $139(67,1)$ & $233(80,0)$ & $372(74,5)$ & $<0,01^{*}$ & $0,50(0,33-0,76)$ \\
Anticoncepcional de & & & & & \\
emergência & $28(13,5)$ & $39(13,3)$ & $67(13,4)$ & $<0,96$ & $1,01(0,60-1,70)$ \\
Espermicida & $65(31,4)$ & $145(49,6)$ & $210(42,0)$ & $<0,01^{*}$ & $0,46(0,31-0,66)$ \\
Calendário & $42(20,3)$ & $50(17,1)$ & $92(18,4)$ & $<0,37$ & $1,22(0,77-1,93)$ \\
Coito interrompido & $85(41,1)$ & $192(65,7)$ & $281(56,3)$ & $<0,01^{*}$ & $0,33(0,23-0,48)$ \\
$\begin{array}{l}\text { Dispositivo } \\
\text { Intrauterino }\end{array}$ & & & & \\
Laqueadura & $29(14,0)$ & $88(30,1)$ & $117(23,4)$ & $<0,01^{*}$ & $0,37(0,23-0,59)$ \\
Vasectomia & $80(38,6)$ & $131(44,8)$ & $211(42,3)$ & $<0,15$ & $0,76(0,53-1,10)$ \\
\hline
\end{tabular}

Fonte: Elaboração própria.

$* \mathrm{p} \leq 0,05$.

Os participantes do sexo masculino apresentavam menor conhecimento a respeito das IST e da prática de sexo seguro (Tabela 2). Quanto à crença de que o contraceptivo oral previne contra IST, observou-se que ela é mais recorrente em adolescentes do sexo masculino $(24,6 \%)$ do que entre os do sexo feminino (17,1\%) ( $\mathrm{p}=0,04)$. Em relação ao uso de preservativo em todas as relações sexuais, observou-se que a maior proporção de adolescentes que julgava a prática desnecessária era do sexo masculino $(22,7 \%)$ em oposição aos de sexo feminino $(9,9 \%)(\mathrm{p}<0,01)$.

Tabela 2 - Percepções dos adolescentes a respeito de sexo seguro, contracepção e participação em atividades educativas sobre sexualidade. Pouso Alegre, Minas Gerais, Brasil - 2017

\begin{tabular}{l|c|c|c|c|c}
\hline Variável & $\begin{array}{c}\text { Masculino } \\
\mathbf{N = 2 0 7}(\%)\end{array}$ & $\begin{array}{c}\text { Feminino } \\
\mathbf{N = 2 9 2}(\%)\end{array}$ & $\begin{array}{c}\text { Total } \\
\mathbf{N = 4 9 9} \\
\mathbf{( \% )}\end{array}$ & p-valor & $\begin{array}{c}\text { Odds Ratio } \\
\text { (Mínino- } \\
\text { Máximo) }\end{array}$ \\
\hline $\begin{array}{l}\text { Acredita que o contraceptivo } \\
\text { oral previne contra infecções } \\
\text { sexualmente transmissíveis }\end{array}$ & & & & $0,04^{*}$ & $1,58(1,02-2,45)$ \\
$\quad$ Sim & $51(24,6)$ & $50(17,1)$ & $101(20,2)$ \\
Não & $156(75,4)$ & $242(82,9)$ & $398(79,8)$
\end{tabular}


Tabela 2 - Percepções dos adolescentes a respeito de sexo seguro, contracepção e participação em atividades educativas sobre sexualidade. Pouso Alegre, Minas Gerais, Brasil - 2017

(conclusão)

\begin{tabular}{|c|c|c|c|c|c|}
\hline Variável & $\begin{array}{l}\text { Masculino } \\
\mathbf{N}=207(\%)\end{array}$ & $\begin{array}{c}\text { Feminino } \\
\mathrm{N}=292(\%)\end{array}$ & $\begin{array}{c}\text { Total } \\
\mathbf{N}=499 \\
(\%)\end{array}$ & p-valor & $\begin{array}{l}\text { Odds Ratio } \\
\text { (Mínino - } \\
\text { Máximo) }\end{array}$ \\
\hline \multicolumn{4}{|l|}{$\begin{array}{l}\text { Acredita que é necessário usar } \\
\text { preservativo em toda relação } \\
\text { sexual }\end{array}$} & $<0,01^{*}$ & $0,37(0,22-0,62)$ \\
\hline $\operatorname{Sim}$ & $160(77,3)$ & $263(90,1)$ & $423(84,8)$ & & \\
\hline Não & $47(22,7)$ & $29(9,9)$ & $76(15,2)$ & & \\
\hline $\begin{array}{l}\text { Diálogo sobre sexualidade com } \\
\text { os pais/responsáveis }\end{array}$ & & & & 0,75 & $0,94(0,65-1,35)$ \\
\hline Sim & $85(41,1)$ & $124(42,5)$ & $209(41,9)$ & & \\
\hline Não & $122(58,9)$ & $168(57,5)$ & $290(58,1)$ & & \\
\hline $\begin{array}{l}\text { Diálogo sobre sexualidade com } \\
\text { os amigos }\end{array}$ & & & & 0,48 & $0,86(0,57-1,30)$ \\
\hline Sim & $151(72,9)$ & $221(75,7)$ & $72(74,5)$ & & \\
\hline Não & $56(27,1)$ & $71(24,4)$ & $127(25,5)$ & & \\
\hline $\begin{array}{l}\text { Importância da realização } \\
\text { de atividades e projetos de } \\
\text { educação sexual nas escolas }\end{array}$ & & & & 0,26 & $0,71(0,39-1,28)$ \\
\hline Sim & $182(88,3)$ & $268(91,5)$ & $450(90,1)$ & & \\
\hline Não & $24(11,6)$ & $24(8,5)$ & $48(9,8)$ & & \\
\hline $\begin{array}{l}\text { Participação em oficinas sobre } \\
\text { sexualidade na escola }\end{array}$ & & & & 0,45 & $0,87(0,61-1,24)$ \\
\hline $\operatorname{Sim}$ & $92(44,4)$ & $151(51,7)$ & $243(48,4)$ & & \\
\hline Não & $115(55,5)$ & $141(48,3)$ & $256(51,6)$ & & \\
\hline
\end{tabular}

Fonte: Elaboração própria.

$* \mathrm{p} \leq 0,05$.

Informações sobre Infecções Sexualmente Transmissiveis (IST) e gravidez

Apesar de a maioria dos participantes $(90,1 \%)$ ter aprovado a realização de atividades e projetos a respeito da sexualidade no ambiente escolar, apenas $48,4 \%$ deles relataram ter participado de oficinas de educação sexual na escola, e também de estabelecer maior diálogo a respeito da sexualidade com amigos (74,5\%) em detrimento de fazê-lo com pais/responsáveis (41,9\%). Não houve diferença em relação ao sexo, quanto ao estabelecimento do diálogo com a família ou amigos ( $\mathrm{p}>0,05)$.

Em relação ao desejo de exercer a maternidade/paternidade em alguma etapa do ciclo vital, a maioria dos adolescentes ( $\mathrm{N}=252 ; 50,5 \%)$ relatou esse desejo, enquanto uma proporção menor apenas cogitou a possibilidade $(\mathrm{N}=184$;
$36,9 \%), 8,2 \%(\mathrm{~N}=41)$ deles relataram que nunca gostariam de ser pais, $0,6 \%(\mathrm{~N}=3)$ não responderam à questão. A experiência de gravidez na adolescência foi referida por $3,8 \%(\mathrm{~N}=19)$ das adolescentes.

\section{Discussão}

A presente casuística detectou que os adolescentes apresentavam algum conhecimento sobre os métodos contraceptivos existentes e disponíveis, no entanto houve diferenças quanto à variedade de métodos referidos entre os adolescentes e as adolescentes. Os métodos de barreira e os hormonais foram os mais citados por ambos os sexos. As adolescentes mostraram algum conhecimento sobre métodos comportamentais, sendo este um diferencial sobre os adolescentes do sexo masculino. 
Entre adolescentes, a principal lacuna de conhecimento ocorre para os itens referentes aos métodos contraceptivos hormonais ${ }^{(10)}$ e ao preservativo masculino $^{(11)}$, resultado que concorda com esta pesquisa, na qual os itens com maior percentual de ignorância ou erros foram aqueles relacionados ao uso de pílula anticoncepcional. Embora o preservativo masculino seja o principal método conhecido pelos adolescentes $(94,4 \%)$, sua eficácia depende do conhecimento da técnica e, sobretudo, da disciplina do usuário. No entanto, circunstancialmente, essa não é a realidade do comportamento sexual do adolescente $^{(12)}$.

Quanto ao conhecimento sobre os métodos comportamentais, é importante que os adolescentes tenham informações sobre a eficácia e indicação, embora, devido ao ciclo menstrual irregular, à dificuldade de manter abstinência sexual periódica e por não apresentar proteção contra IST, esses métodos não são inicialmente recomendados para todos os adolescentes ${ }^{(11,13)}$.

Além das diferenças entre os distintos métodos contraceptivos, outro resultado desta investigação foi a diferença de conhecimento entre os adolescentes e as adolescentes quanto à prática do sexo seguro tanto para a prevenção da gravidez quanto para evitar a transmissão de IST. As adolescentes mostraram maior conhecimento quanto à importância do uso de preservativo para a prevenção de IST em relação aos adolescentes do sexo masculino. A maior proporção dos adolescentes que julgavam desnecessário o uso do preservativo em todas as relações sexuais era do sexo masculino, grupo que também apresentou o conhecimento equivocado de que os contraceptivos hormonais previnem contra IST.

Tal contexto de conhecimentos insuficientes ou inadequados não é exclusividade do cenário de pesquisa deste artigo. Estudo realizado na região Sul do Brasil, por exemplo, detectou que $37,1 \%$ dos adolescentes do sexo feminino e $30,5 \%$ daqueles do sexo masculino entendiam que contraceptivos orais poderiam ser usados também como método preventivo para IST ${ }^{(14)}$.

Outro estudo, realizado em Bokeo Province, Lao, mostrou que, em média, os adolescentes conheciam vários métodos contraceptivos tradicionais. Os mais conhecidos eram o preservativo masculino e a pílula $(60,2 \%$ e 51,5\%, respectivamente). Aproximadamente um terço $(32,4 \%)$ dos adolescentes desta pesquisa não conhecia nenhum anticoncepcional moderno. Embora mais meninos conhecessem o preservativo masculino ou a pílula (19,7\% dos meninos, em comparação com 12,2\% das meninas), os níveis de conhecimento não variaram significativamente entre os $\operatorname{sexos}^{(15)}$.

A contracepção dupla, caracterizada pelo uso concomitante da pílula e do preservativo, embora recomendado contra IST e gravidez, não tem aceitação suficiente entre os adolescentes ${ }^{(11)}$. Estudo desenvolvido em Missouri, Estados Unidos, sobre contracepção dupla em mulheres adolescentes e adultas encontrou que 45\% daquelas que não utilizavam métodos contraceptivos de longa duração usavam dupla contracepção ${ }^{(16)}$. Independentemente do método escolhido, os adolescentes devem ser aconselhados sobre a importância do uso de preservativos para reduzir o risco de aquisição de IST, caracterizando a contracepção dupla ${ }^{(17)}$. Outro estudo mostra que o preservativo masculino é o método mais conhecido contra IST e gravidez entre os jovens, apesar do seu uso consistente não ser frequente, principalmente nas relações eventuais e não programadas ${ }^{(2)}$.

A escolha do método contraceptivo é influenciada por vários fatores, incluindo o acesso a diferentes métodos, características pessoais do adolescente, do seu parceiro e da tecnologia disponível. O conhecimento sobre vários métodos contraceptivos e o desejo de usá-los são essenciais para o sucesso da contracepção ${ }^{(11)}$. Outro apontamento deste estudo destaca que 15,2\% dos adolescentes não desejavam usar o preservativo em toda relação sexual, no entanto 90,1\% deles mostraram interesse em adquirir mais conhecimento sobre educação sexual nas escolas.

Chama a atenção o fato de os adolescentes não terem citado métodos contraceptivos reversíveis de longa duração, como os implantes e o dispositivo uterino com hormônio. Este resultado também instiga a propor-se outros estudos, 
considerando não apenas o conhecimento dos adolescentes, mas as expectativas e possibilidades de maior e mais fácil acesso aos métodos contraceptivos considerados seguros e adequados para o adolescente.

Este estudo mostrou que os participantes apresentavam lacunas no conhecimento a respeito dos métodos contraceptivos e na prevenção de IST, bem como que eles viam como profícua a participação em atividades educativas a respeito do tema. O estudo ainda identificou que a maior parte dos adolescentes estabelecia mais comunicação com amigos do que com os pais. É importante aproveitar oportunidades de palestras em escolas, para promover o conhecimento e encorajar a adoção de hábitos e práticas sexuais saudáveis, que assegurem riscos mínimos à saúde sexual e reprodutiva de adolescentes $^{(18)}$. Informação e comunicação devem ser duas aliadas na prevenção e na promoção da saúde, evitando a disseminação de informações equivocadas.

Apesar de reconhecerem a importância da escola na educação sexual, os adolescentes também citaram a corresponsabilidade dos pais e a relevância da participação familiar no tema. Interessante notar que, contraditoriamente, o papel do diálogo com a família a respeito da sexualidade ainda é difícil de ser estabelecido ${ }^{(2)}$.

Ressalta-se que as adolescentes estabeleciam maior diálogo sobre sexualidade com as mães, em relação aos adolescentes do sexo masculino, entretanto o assunto é pouco abordado com a figura paterna tanto pelos adolescentes do sexo feminino quanto do masculino ${ }^{(14)}$. No serviço de saúde, a consulta médica ou de enfermagem pode configurar-se como ocasião oportuna de promoção da saúde e educação em saúde, quando o momento de interação entre profissional de saúde e adolescente é utilizado para esclarecimento de dúvidas e oferta de informações qualificadas sobre a sexualidade ${ }^{(19)}$.

Observa-se uma redução nas atividades de orientação para prevenção de gravidez nas escolas $^{(20)}$. Neste estudo, por exemplo, metade da amostra relatou não ter participado de nenhuma atividade de educação sexual no ambiente escolar. Fica evidente, portanto, que o planejamento estratégico de ações sistematizadas de educação em saúde é fundamental para a promoção da saúde sexual e reprodutiva dos adolescentes. Oficinas de educação sexual auxiliam não apenas na compreensão dos riscos da gravidez na adolescência, como também do risco de contrair IST ${ }^{(21)}$. Desse modo, a escola e o Estado deveriam trabalhar juntos para contemplar a educação sexual enquanto tema transversal nos currículos escolares ${ }^{(2)}$, bem como realizarem a interlocução com pais e familiares, buscando o estabelecimento de uma rede de apoio social.

Os resultados do presente estudo fornecem dados que subsidiam discussões no contexto da promoção da saúde do adolescente, demonstram a necessidade da orientação contraceptiva e da prevenção de IST para os adolescentes, e a importância da sensibilização da participação masculina nas discussões, tendo em vista o maior déficit de conhecimento entre os adolescentes do sexo masculino.

É pertinente apontar que, durante o percurso para concretização do estudo, houveram limitações, uma delas associada à anuência dos pais/responsáveis para participação dos alunos na pesquisa. Os TCLE que eram levados para a casa, acabavam esquecidos seja por não conversarem com os pais sobre a necessidade de serem assinados, seja por não encontrarem os pais em casa, seja por terem perdido o termo. Enfim, houve certa dificuldade e consequente perda de alunos que não devolveram os termos e, assim, não puderam participar da pesquisa. Outro ponto esteve associado à metodologia ter sido desenhada e realizada em forma de aplicação de questionários, obtendo-se informações de forma direta, o que pode ter comprometido a identificação de questões mais amplas e maior entendimento sobre os comportamentos e conceitos pré-estabelecidos. Considerou-se também como limitação a influência de elementos comportamentais dos pesquisados, fazendo com que não respondessem com veracidade as questões abordadas, devido a vergonha, timidez, falta de entendimento ou do estigma associado ao tema. 


\section{Conclusão}

Pôde-se concluir que os estudantes apresentaram concepções prévias e eventualmente superficiais a respeito da prevenção de IST e da gravidez na adolescência. Os participantes do sexo masculino apresentaram menor conhecimento a respeito dos métodos contraceptivos oferecidos, IST e prática do sexo seguro.

Ao considerar a necessidade de aprimorar o conhecimento dos adolescentes a respeito da saúde sexual, ressalta-se a pertinência da realização de atividades educativas a respeito da promoção da saúde sexual e reprodutiva no âmbito escolar. Essas ações devem valorizar o empoderamento das adolescentes do sexo feminino, além da participação ativa e inclusão dos adolescentes do sexo masculino, com vistas a atingir a equidade de gênero, sensibilização, reflexão e corresponsabilização de ambos os sexos na prática do sexo seguro e saudável.

A realização de atividades educativas isoladas apresenta pouco impacto na formação de conceitos e atitudes dos adolescentes. A promoção do autocuidado e da prática do sexo seguro, reforçando a responsabilidade quanto ao exercício da sexualidade com saúde, devem ser consideradas, reforçando a prática de comportamentos e atitudes para uma vida sexual saudável. Considera-se que a abordagem da educação sexual, por ser um tema amplo e complexo, deveria ser realizada de forma contínua, em articulação com as escolas, as famílias e a comunidade, além de envolver todos os elementos que compõem os equipamentos da rede de apoio social aos adolescentes.

\section{Colaborações:}

1 - concepção, projeto, análise e interpretação dos dados: Kleber José Vieira, Nayara Gonçalves Barbosa, Juliana Cristina dos Santos Monteiro, Letícia de Almeida Dionízio, Flávia Azevedo Gomes-Sponholz;

2 - redação do artigo e revisão crítica relevante do conteúdo intelectual: Kleber José Vieira, Nayara Gonçalves Barbosa, Juliana
Cristina dos Santos Monteiro, Letícia de Almeida Dionízio, Flávia Azevedo Gomes-Sponholz;

3 - aprovação final da versão a ser publicada: Kleber José Vieira, Nayara Gonçalves Barbosa, Juliana Cristina dos Santos Monteiro, Letícia de Almeida Dionízio, Flávia Azevedo Gomes-Sponholz.

\section{Referências}

1. Anderson LE, Dingle GA, O'Gorman B, Gullo MJ. Young adults' sexual health in the digital age: perspectives of care providers. Sex Reprod Health. 2020;25:100534. DOI: 10.1016/ j.srhc. 2020.100534

2. Almeida RAAS, Corrêa RGCF, Rolim ILTP, Hora JM, Linard AG, Coutinho NPS, et al. Conhecimento de adolescentes relacionados às doenças sexualmente transmissíveis e gravidez. Rev Bras Enferm. 2017;70(5):1087-94. DOI: https:// doi.org/10.1590/0034-7167-2016-0531

3. Israni ST, Matheny ME, Matlow R, Whicther D. Equity, inclusivity, and innovative digital technologies to improve adolescent and young adult health. J Adolesc Health. 2020;67(2):S4-S6. DOI: $10.1016 /$ j.jadohealth.2020.05.014

4. Brasil. Ministério da Saúde. Proteger e cuidar da saúde de adolescentes na atenção básica. Brasília (DF); 2017 [cited 2019 Jul 14]. Available from: http://bvsms.saude.gov.br/bvs/publicacoes/ proteger_cuidar_adolescentes_atencao_basica.pdf

5. Maness SB, Buhi ER, Daley EM, Baldwin JA, Kromrey JD. Social determinants of health and adolescent pregnancy: an analysis from the National Longitudinal Study of Adolescent to Adult Health. J Adolesc Health. 2016;58(6):636-43. DOI: 10.1016/j.jadohealth.2016.02.006

6. Leftwich HK, Alves MVO. Adolescent pregnancy. Pediatr Clin North Am. 2017;64(2):381-8. DOI: 10.1016/j.pcl.2016.11.007

7. Wendland EM, Horvath JDC, Kops NL, Bessel M, Caierão J, Hohenberger GF, et al. Sexual behavior across the transition to adulthood and sexually transmitted infections: findings from the national survey of human papillomavirus prevalence (POP-Brazil). Medicine. 2018;97(33):e11758. DOI: 10.1097/MD.0000000000011758

8. Brasil. Ministério da Educação. Secretaria de Educação Fundamental. Parâmetros curriculares nacionais: pluralidade cultural, orientação sexual. 
Brasília (DF); 1997 [cited 2020 Feb 18]. Available from: http://portal.mec.gov.br/seb/arquivos/pdf/ livro101.pdf

9. Brasil. Ministério da Saúde. Pesquisa de conhecimento, atitudes e práticas na população brasileira. Brasília (DF); 2011 [cited 2020 Feb 20]. Available from: http://www.aids.gov.br/pt-br/ pub/2016/pesquisa-de-conhecimentos-atitudes-epraticas-na-populacao-brasileira-pcap-2013

10. Sanz-Martos S, López-Medina IM, Álvarez-García C, Álvarez-Nieto C. Sexuality and contraceptive knowledge in university students: instrument development and psychometric analysis using item response theory. Reprod Health. 2019;16(1):127. DOI: 10.1186/s12978-019-0791-9

11. Apter D. Contraception options: aspects unique to adolescent and young adult. Best Pract Res Clin Obstet Gynaecol. 2018;48:115-27. DOI: 10.1016/j.bpobgyn.2017.09.010

12. Costa GPO, Guerra AQS, Araújo ACPF. Conhecimentos, atitudes e práticas sobre contracepção para adolescentes. J res: fundam care. 2016;8(1):3597-608. DOI: 10.9789/21755361.2016.v8i1.3597-3608

13. Bitzer J, Abalos V, Apter D, Martin R, Black A, Global Care (Contraception: Access, Ressouces, Education) Group. Targeting factors for change: contraceptive counselling and care of female adolescents. Eur J Contracept Reprod Health Care. 2016;21(6):417:30. DOI: 10.1080/13625187.2016.1237629

14. Genz N, Meincke SMK, Carret MLV, Corrêa ACL, Alvez C.N. Doenças sexualmente transmissíveis: conhecimento e comportamento sexual de adolescentes. Texto Contexto Enferm. 2017;26(2):1-12. DOI: 10.1590/0104-07072017005 100015
15. Phongluxa K, Langeslag G, Jat TR, Kounnavong S, Khan MA, Essink DR. Factors influencing sexual and reproductive health among adolescents in Lao PDR. Glob Health Action. 2020; 3(sup2):28-37. DOI: $10.1080 / 16549716.2020 .1791426$

16. Bernard C, Zhao Q, Peipert JF. Dual method use among long-acting reversible contraceptive users. Eur J Contracept Reprod Health Care. 2018;23(2):97-104. DOI: 10.1080/13625187. 2018.1445850

17. Todd N, Black A. Contraception for adolescents. J Clin Res Pediatr Endocrinol. 2020; 12(Suppl 1):28-40. DOI: $10.4274 /$ jcrpe.galenos. 2019.2019.S0003

18. Franco MS, Barreto MTS, Carvalho JW, Silva PP, Moreira WC, Cavalcante MC et al. Sexual and reproductive health education of adolescent students. Rev enferm UFPE on line. 2020;14:1-8. DOI: 10.5205/1981-8963.2020.244493

19. Eisenstein E. Medicina e saúde de adolescentes: o octaedro de ações. Adolesc Saude [Internet]. 2018 [cited 2020 Ago 14];15(1):21-33. Avaliable from: https://cdn.publisher.gn1.link/adolescenciae saude.com/pdf/v15s1a04.pdf

20. Felisbino-Mendes MS, Paula TF, Machado IE, Oliveira-Campos M, Malta DC. Análise dos indicadores de saúde sexual e reprodutiva de adolescentes brasileiros, 2009, 2012 e 2015. Rev Bras epidemiol. 2018;21:1-14. DOI: 10.1590/1980549720180013.supl.1

21. França MTA, Frio GS. Factors associated with family, school and behavioral characteristics on sexual initiation: a gender analysis for Brazilian adolescents. PLoS One. 2018;13(12):1-16. DOI: 10.1371/journal.pone. 0208542

Recebido: 26 de setembro de 2020

Aprovado: 12 de janeiro de 2021

Publicado: 10 de fevereiro de 2021

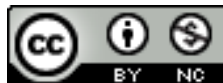

A Revista Baiana de Enfermagem utiliza a Licença Creative Commons - Atribuição-NãoComercial 4.0 Internacional. https://creativecommons.org/licenses/by-nc/4.0/ Este artigo é de acesso aberto distribuído sob os termos da Licença Creative Commons (CC BY-NC). Esta licença permite que outros remixem, adaptem e criem a partir do seu trabalho para fins não comerciais. Embora os novos trabalhos tenham de lhe atribuir o devido crédito e não possam ser usados para fins comerciais, os usuários não têm de licenciar esses trabalhos derivados sob os mesmos termos. 